\title{
ESTUDIO GRANADA SOBRE RETINOPATÍA DIABÉTICA. CRIBADO DE 8.244 PACIENTES DIABÉTICOS. I
}

\section{THE GRANADA DIABETIC RETINOPATHY STUDY. DIRECT SCREENING OF 8,244 PATIENTS. I}

\author{
GARCÍA-SERRANO JL ${ }^{1}$, SERRANO-LABORDA D ${ }^{1}$, LÓPEZ-POZAS M² ${ }^{2}$ CABELLO-APARICIO C ${ }^{2}$, \\ CASTRO-ROSALES L ${ }^{3}$
}

\section{RESUMEN}

Objetivo: Evaluar el programa de cribado regular de la retinopatía diabética en la población asistida del Hospital Universitario San Cecilio de Granada. Material y métodos: Se realizó durante un seguimiento de 2 años, un estudio de cohortes prospectivo y multicentrico. El estudio comprende las exploraciones de la retina de 8.244 diabeticos en 14 centros de salud. Se realizaron 11.924 exámenes de retina en midriasis utilizando un oftalmoscopio indirecto.

La clasificación de las lesiones fue la recomendada por la Escala Internacional Simplificada de Retinopatia Diabética y Edema Macular. La periocidad de las exploraciones fueron las recomendadas por el ETDRS.

Resultados: La prevalencia de diabetes en el area de salud fue del $2,77 \%$. La asistencia media al programa fue del $84,1 \%$, solamente en un $0,3 \%$ de los pacientes el fondo de ojo fue inexplorable.

El 91,3\% de la poblacion diabética conocida acudió al menos a un examen de fondo de ojo, remitiéndose para tratamiento hospitalario el $3,4 \%$ de los pacientes.

\begin{abstract}
Objectives: To evaluate the results of regular screening for diabetic retinopathy in the geographic area assigned to the San Cecilio University Hospital of Granada.

Material and methods: A multicenter prospective cohort study was carried out over a 2-year followup period. Retinal examinations were performed in 8244 diabetics in 14 health centres. 11,924 retinal examinations using an indirect ophthalmoscope, following pupil dilatation, were carried out.

The classification of the lesions was as recommended by the simplified international scale of diabetic retinopathy and macular edema. The intervals between fundus examinations followed those recommented by the etdrs.

Results: The crude prevalence of diabetes in the health area concerned was $2.77 \%$. The average screening compliance of the program was $84.1 \%$, and in only $0.3 \%$ of the patients was the fundus ophthalmoscopy ungradable.

91.3\% of the known diabetic population attended for at least one fundus examination, with only $3.4 \%$
\end{abstract}

\footnotetext{
Recibido: 24/5/06. Aceptado: 20/9/07.

Hospital Universitario «San Cecilio». Servicio de Oftalmología. Granada. España. Distrito Metropolitano de Granada. Distrito Granada. Ambulatorio del Zaidin (Granada). Ambulatorio de Loja (Granada). Zonas Básicas de Salud de Góngora, Miraflores, Mirasierra, Las Flores, Zaidín Centro y Zaidín Sur, Armilla, Alhama de Granada, Cenes de la Vega, Churriana de la Vega, Huetor Tajar, Montefrío, Valle de Lecrín, Loja (todas en la provincia de Granada). Y los consultorios adscritos a estas zonas básicas de salud.

1 Doctor en Medicina. Hospital Universitario San Cecilio. Granada.

2 Licenciada en Medicina. Hospital Universitario San Cecilio. Granada.

3 Responsable LEMEH. Distrito Granada.

Correspondencia:

José Luis García Serrano

C/. Periodista Miguel Montalvo Jiménez, 6

18014 Granada

España

E-mail: jopalace@ @otmail.com
} 
El costo anual del Programa se cifró en 53.173 euros, con una media de 6.000 exploraciones anuales a un costo de 8.87 euros exploración.

Conclusiones: El programa de cribado de la retinopatía diabética realizado con un oftalmoscopio binocular y en midriasis es costo-efectivo. Proporciona cobertura a toda la población diabética conocida con un elevado grado de cumplimentación. El porcentaje de pacientes cuyo fondo de ojo se considera inexplorable es mínimo.

Palabras clave: Retinopatía diabetica, cribado poblacional, clasificación, diagnóstico, estructura organizativa, equipo, costo.

\section{INTRODUCCIÓN}

En el año 2001, el Servicio Andaluz de Salud define su mapa de de procesos asistenciales como el conjunto de patologías que plantean un reto asistencial y de calidad en la asistencia sanitaria.

En el manual del proceso de diabetes tipo I y tipo II, se recoge la obligación de realizar al menos un fondo de ojo anual a los diabéticos tipo I y al menos uno bianual a los diabéticos tipo II $(1,2)$. Y que esta exploración se realice desde atención primaria mediante un retinógrafo digital.

Entre los años 2003-2007, se planifica y ejecuta el plan integral de la diabetes y el programa de detección de la retinopatía diabética $(3,4)$. Se consigue un desarrollo telemático suficiente para el soporte de imágenes y la realización de un cribado masivo de la retinopatía diabética en Andalucía.

En el año 2003, nuestro servicio no disponía aún de los retinógrafos digitales. En el año 2004 en una editorial de la revista Acta Ophtalmogica (5) llama la atención a la Comunidad Oftalmológica internacional sobre las deficiencias del cribado: «la insuficiente puesta a punto del desarrollo informático no puede ser una excusa para no realizar los programas de cribado masivo de la retinopatía diabética que demandan nuestros pacientes».

La fotocoagulación temprana en la retinopatía diabética puede reducir el porcentaje de ceguera hasta en un $90 \%$. Los programas de cribado masivo pretenden detectar precozmente aquellos pacientes con retinopatía diabética susceptible de tratamiento, antes que las complicaciones aparezcan $(6,7)$.

La prevalencia real de diabetes no es bien conocida en España y en la mayoría de las publicaciones of the patients being referred for hospital treatment. The annual cost of the program was set at 53,173 euros, with the average cost of each examination (6000 per year) being 8.87 euros.

Conclusions: The Diabetic Retinopathy Screening Program (through dilated pupils) carried out with indirect ophthalmoscopy is cost-effective. It provides coverage to the whole known diabetic population with a high level of compliance. The percentage of patients whose fundus can be considered ungradable is minimal (Arch Soc Esp Oftalmol 2007; 82: 681-690).

Key words: Diabetic retinopathy, mass screening, classification, diagnosis, organizational structure, equipment, cost.

se recoge a aquella población diabética más demandante, a menudo la más grave, llevando los cálculos de prevalencia de la retinopatía diabética al alza.

La alta prevalencia de la retinopatía diabética. La alta sensibilidad y especificad obtenida con el oftalmoscopio binocular, entre los pacientes con retinopatía diabética que pueden necesitar tratamiento; nos motivó a la realización de este cribado masivo.

Este artículo recoge la estructura organizativa seguida en nuestro servicio, desde el año 2003, hasta finales del año 2005 en los que se realizó un cribado directo y masivo de la población diabética, de nuestra área de Salud con un ordenador portátil y un oftalmoscopio binocular.

\section{SUJETOS, MATERIAL Y MÉTODOS}

El equipo asistencial estaba compuesto por: un oftalmólogo, un administrativo y un auxiliar de enfermería. El material constaba de un binocular y un ordenador portátil. En el ordenador iba insertado un fichero con la BDU, conjunto básico y mínimo de datos de los enfermos de nuestra área sanitaria.

La asistencia sanitaria del Hospital Universitario «San Cecilio» es de carácter público. En España la cobertura del Seguro Nacional incluye al 95\% de la población. Las compañías privadas generalmente excluyen de su cobertura sanitaria al paciente diabético.

La planificación previa incluía negociar con los dos Distritos Sanitarios, como cabeceras, y con los directores de los centros de salud. Y después realizar un cronograma planificado de visitas. 
Algunos centros de salud desconocían el número de diabéticos que atendían y en algunos casos faltaban datos para su filiación completa. Para incorporar a todos los diabéticos, se abrió un cronograma de citas en cada centro de salud. La información administrativa de la exploración de fondo de ojo se iba contrastando y completando con la obtenida en su centro de salud.

El día asignado eran convocados 80 enfermos en el centro de Salud, con citas correlativas cada 3 minutos desde las 8,30 horas a las 12,30 horas. A las 8,30 horas el auxiliar de enfermería dilataba el primer enfermo con colirio de tropicamida y fenilefrina. Después iba dilatando sucesivamente a los pacientes al tiempo que les asignaba el número de orden que se correspondía con la hora de su cita.

A las 9 horas se atiende al primer enfermo dilatado. El número de la tarjeta permite al administrativo vaciar sus datos desde la BDU (base de datos unificada) en una plantilla previamente diseñada por nosotros (fig. 1), y se crea un nuevo archivo únicamente con los datos de los enfermos diabéticos explorados o que citados no se habían presentado este día.

En la plantilla constaba el ID (número de identificación correlativo que asignaba el ordenador), NUSS (número de Seguridad Social de la Tarjeta sanitaria individual), fecha de nacimiento, edad, dirección del enfermo, número de teléfono, centro de salud y nombre de su médico de familia.

La plantilla presentaba seis campos médicos abiertos: fecha de la exploración, edad de comienzo de la diabetes (año), tratamiento (dieta, antidiabéticos orales, insulina), tipo de retinopatía (no retinopatía, leve, moderada, severa, proliferativa, edema macular, fotocoagulación, no asiste), nueva cita (número de meses) y observaciones. Los cinco primeros campos están estructurados de tal modo que

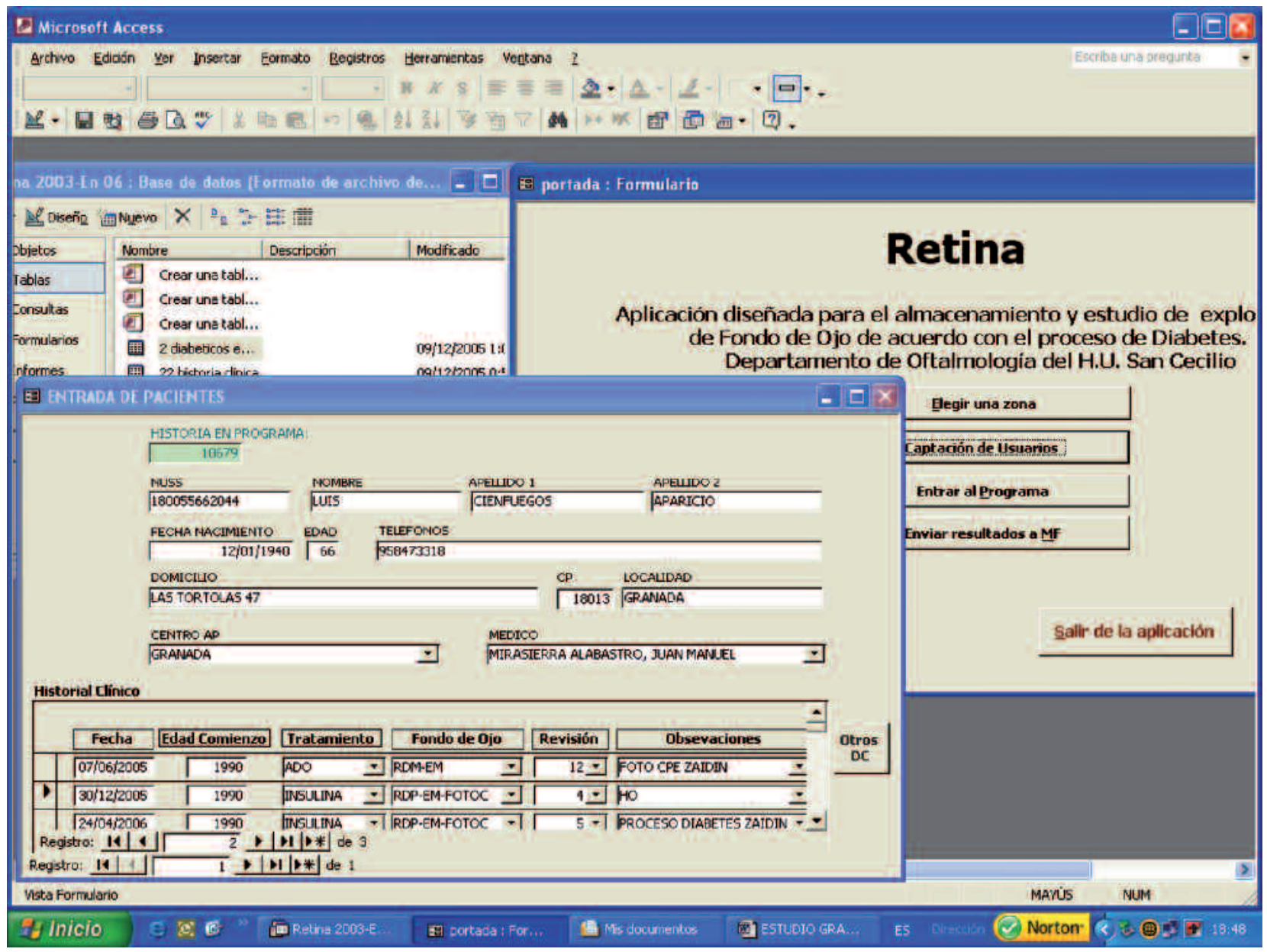

Fig. 1: Datos administrativos y clínicos de un paciente simulado con retinopatía diabética. 
sólo es necesario pinchar la opción correcta del menú desplegable para adicionar el dato.

Primero se registran los datos administrativos, después el enfermo pasa a un sillón sencillo y cómodo donde se le explora con el oftalmoscopio binocular y se valora su fondo de ojo. Al tiempo que se explora el paciente, el administrativo incluye los datos sanitarios: tipo de retinopatía diagnosticada en el paciente, cuando se realizará su próxima revisión y si precisa ser enviado al hospital por patología diabética sugerente de tratamiento y a qué unidad irá destinado.

Se ha utilizado la escala internacional simplificada para clasificar la retinopatía diabética y el edema macular del año 2003 (8) que creemos se adapta mejor que la clasificación ETDRS (9) para el cribado masivo de población diabética. Anomalías venosas y anomalías microvasculares intraretinianas son difíciles de reconocer en ausencia de una buena retinografía, lo que hace difícil de aplicar la escala ETDRS por su complejidad.

La clasificación ETDRS aunque esta considerada el «Gold standard», sin embargo no se utiliza en la clínica porque presenta muchos niveles y estadios, requiere fotógrafos experimentados y un equipo sofisticado de almacenamiento y archivo y no ha probado ser fácil ni práctica $(10,11)$. La clasificación simplificada se apoya en la evidencia científica de la clasificación ETDRS y en sus niveles. Esta clasificación solo pretende un manejo más sencillo en la práctica clínica $(10,11)$. Ha sido asumida por la Academia Americana de Oftalmología como medio de unificar la terminología en los manuscritos y comparar resultados entre distintos estudios $(11,12)$; sin embargo, todavía no existen evidencias sobre su utilidad y aceptación. Algunos autores en el ámbito hospitalario prefieren la clasificación ETDRS porque lleva asociado un pronóstico visual (13).

Periódicamente cada mes son enviados a los centros de salud, los listados de los enfermos explorados dirigidos al director del Centro y desglosados por los médicos de familia que atienden a cada grupo de enfermos explorados. Este listado incluye el resultado de la exploración; el nombre del enfermo, la dirección, su médico de atención primaria, el resultado del fondo de ojo y la fecha aproximada de su próxima cita.

La base de datos que manejamos en Access consta de tres plantillas: una plantilla para captar el enfermo por nombre y apellidos o número de Seguridad Social. Una segunda plantilla en blanco por si el paciente no estuviera incluido en la BDU y para poder introducir manualmente todos los datos. Una tercera plantilla donde queda recogido el resultado de la exploración.

La periodicidad en la exploración de fondo de ojo fue la siguiente: 1. Se exploró bianualmente a los pacientes con diabetes mellitus de menos de 10 años de evolución, no insulinodependientes y con buen control glucémico. 2. Se exploró anualmente: a) a los pacientes con retinopatía diabética leve y buen control glucémico. b) A los pacientes con diabetes mellitus tipo I. c) A los pacientes en tratamiento con insulina. 3. Se exploró cada 4 meses o menos a los pacientes con retinopatía diabética moderada, severa, proliferativa o con edema macular.

\section{RESULTADOS}

Los datos de este trabajo proceden de una cohorte dinámica multicéntrica, constituida por todos los diabéticos que se han podido identificar en nuestra área Sanitaria de unos 298.000 habitantes y son el resultado de 11.924 exploraciones realizadas a 8.244 diabéticos, entre noviembre de 2003 y diciembre de 2005.

La prevalencia de diabetes conocida en nuestra área de Salud es del 2,77\% (8244/298.000). Tienen 19 años o menos el $0,75 \%$ de los diabéticos, de 20 a 79 años son el $85,32 \%$ de nuestra muestra y de 80 años o más el 13,93\%. El 2,36\% de nuestra población asistida corresponde a diabéticos entre 20 y 79 años $(2,77 \%$ x 0,8532).

Los diabéticos de 39 años o menos son el 4,08\% de la muestra, y de 40 años o más el 95,92\% de total. Los diabéticos mayores de 40 años constituyen el $2,66 \%$ de nuestra población general $(2,77 \mathrm{x}$ 0,9592).

El fondo de ojo en midriasis se consideró inexplorable únicamente en el $0,3 \%$ de los pacientes (26/8.244). En el 99.7\% de los diabéticos se pudo valorar al menos un ojo.

Oclusión venosa retiniana: Se encontró en el 0,17\% del total (14/8244). En ocasiones fue la única lesión de fondo de que encontramos y en 12 de ellos fue el motivo de derivación al Hospital.

No diabéticos: Se halló en 14 pacientes de 8.256, el $0,17 \%$. Bien porque el diagnóstico aún no era definitivo o porque los pacientes se presentaron al programa buscando el diagnóstico de otra patología. 
Exitus: Los familiares o los registros del sistema sanitario permitieron detectar el exitus únicamente en seis pacientes.

Número de revisiones en función del número de años de evolución de la diabetes. El número de consultas en los pacientes diagnosticados más recientemente, entre los años 2003 a 2006, no se corresponde con su asistencia a las cita. Y ello se produce porque muchos pacientes todavía no se consideran diabéticos. Así partimos desde el año 2002 con un número máximo de revisiones (tabla I).

Se halló una reducción anual del $3 \%$ en el número de revisiones a medida que aumenta el número de años de evolución de la diabetes.

Las renuncias voluntarias al programa. Se obtienen en nueve pacientes de un total de 8.244. Aquí puede quedar enmascarada también un porcentaje elevado de falta de asistencia, con renuncia al programa no declarada.

Glaucoma por cierre angular unilateral. Se presentó en un paciente a las dos horas de la dilatación. Acudió de nuevo al centro de salud donde se estaba realizando el programa y se envió al hospital. A las tres horas del inicio del cuadro, el glaucoma estaba resuelto con la realización de una iridotomía láser YAG.

Cumplimiento de cribado de retinopatía diabética. Del total de 11.924 revisiones proyectadas, se realizaron finalmente 9.652 exploraciones. Ello representó un porcentaje de asistencia total del $81,44 \%$ y una media de no asistencia a la citación del $18,56 \%$.

Tabla I. A más años de evolución de la diabetes, se observa un menor número de exploraciones de fondo de ojo realizadas

Se diagnóstico Número de revisiones Años de evolución la diabetes

\begin{tabular}{rcr}
\hline Año 2002 & 508 & 3 \\
1998 & 400 (un 20\% menos) & 7 \\
1996 & 350 (un 30\% menos) & 10 \\
1990 & 211 (un 56\% menos) & 16 \\
1985 & 159 (un 68\% menos) & 21 \\
1976 & 56 (un 89\% menos) & 30 \\
\hline \hline
\end{tabular}

Las sucesivas citas ha permitido la incorporación progresiva de los diabéticos al programa. El 91,3\% de nuestra población diabética conocida ha sido explorada al menos una vez. Sin embargo el 8,7\% de los diabéticos conocidos no ha acudido a ninguna cita.

En la tabla II se observa una ligera reducción en la asistencia entre aquellos que tienen una retinopatía diabética leve (faltan un 5\% más que aquellos que no tienen retinopatía diabética). Y también una ligera reducción entre aquellos que presentan un grado más avanzado de retinopatía (de 20 posibles citas faltarían a dos más que aquellos que no tienen retinopatía diabética).

En el año 2003 se les realizó a 432 pacientes un examen de fondo de ojo y el grueso de las exploraciones se realizó en los años: 2004 y 2005: 5.913 y 5.579 exámenes.

A pesar de una cuidadosa planificación, el número de enfermos citados presentó una gran disparidad. Se halló una media de 65 enfermos citados en cada centro de salud, de ellos faltaban a la cita unos 13 pacientes (el 18,4\%). La cifra media de enfermos explorados cada día era de 52 .

Se realizó un desglose de exploraciones y citas en los centros de salud en la tabla II. La cifra media de enfermos explorados cada día osciló entre 50 y 70; excepto en dos centros (uno con 35 y otro con 99). Lo que sí se encontró es una gran disparidad en el número diario de enfermos citados en un mismo centro de salud, algunos días se exploraron a más de 100 , y otros exploramos a menos de 30 pacientes (tabla III).

El cálculo realizado sobre el coste anual del cribado de la retinopatía diabética, incluye el coste de una consulta de atención especializada, a la que se adicionan los costes: del administrativo, el auxiliar clínico y de las llamadas telefónicas. El costo generado por día de exploración fue de unos 590,8 Euros. Si el número medio anual de exploraciones realizadas fue de 90 . El coste total anual de programa es de 53.173 euros. Si se realizan 6.000 exploraciones anuales, el costo final de cada exploración de fondo de ojo es de 8,87 euros/paciente/año.

Tabla II. Número de pacientes y el porcentaje de los mismos, que no asisten a la cita en relación al grado de retinopatía diabética

Acuden al cribado

No retinopatía diabética Retinopatía diabética leve Ret. Diab. Mod+Sev+Proli.
$87,96 \%(7713 / 8769)$

$82,1 \%(859 / 1047)$

$77,48 \%(1080 / 1394)$
No cumplen la cita

$12,04 \%(1056 / 8769)$

$17,9 \%(188 / 1047)$

$22,52 \%(314 / 1394)$ 
Tabla III. Número de revisiones realizadas y número medio de diabéticos citados a explorar en cada uno de los Centros de Salud

\begin{tabular}{lccc}
\hline Centros de Salud & N. $^{\circ}$ de exploraciones & N. ${ }^{\circ}$ medio enfermos & N. ${ }^{\circ}$ Mínimo y Máximo \\
\hline Alhama & 8 & 76 & 42 a 136 \\
Armilla & 20 & 82 & 22 a 166 \\
Cenes de la Vega & 8 & 52 & 17 a 102 \\
Churriana & 10 & 99 & 76 a 167 \\
Las Flores & 10 & 35 & 17 a 74 \\
Góngora & 14 & 46 & 9 a 64 \\
Huetor Tajar & 13 & 77 & 8 a 137 \\
Loja & 14 & 50 & 18 a 71 \\
Mirasierra & 14 & 59 & 10 a 105 \\
Montefrío & 7 & 77 & 21 a 140 \\
Zaidin Centro & 9 & 72 & 34 a 107 \\
Zaidin Sur & 18 & 74 & 10 a 122 \\
La Zubia & 28 & 59 & 13 a 178 \\
Valle de Lecrin & 11 & 52 & 7 a 105 \\
\hline \hline
\end{tabular}

\section{DISCUSIÓN}

En el año 2005, la Federación Internacional de diabetes de la región europea estima para España una prevalencia de diabetes del $10,1 \%$ en la población de entre 20 y 79 años. Cuando se consideran estas edades, la prevalencia de diabetes en nuestra población se encontró un porcentaje del 2,77\%. Las cifras presentadas por el IDF-E pueden estar infladas un $365 \%$ respecto a los datos reales de nuestra población asistida.

Para conocer la prevalencia de retinopatía diabética en la población se han intentado distintas estrategias: 1. Explorar a los primeros pacientes diabéticos que acudan a la consulta de su médico de atención primaria (14). 2. Realizar un muestreo aleatorio de centros de salud e incluir a los primeros pacientes diabéticos que acuden al centro de salud (15). 3. Obtener una muestra, de los archivos, de los pacientes diabéticos visitados regularmente (16).

Nosotros acudimos a todos los centros de salud y a los consultorios de nuestra área sanitaria para conocer la prevalencia real de nuestra población asistida. Se citaron a todos los diabéticos conocidos y se incorporó al examen a los diabéticos que se diagnosticaron por primera vez entre los años 2003 y 2005 . La prevalencia poblacional fue del $2,77 \%$.

Kempen 2004 (17) publica que en la población norteamericana de más de 40 años, el 3,55\% son diabéticos. En este rango, nosotros hallamos una prevalencia de diabetes del $2,66 \%$ en nuestra población. Romero-Aroca encuentra una prevalencia del $2,12 \%$ en su población general (16).
Si se quiere reducir la pérdida de visión asociada a la retinopatía diabética, la realización del cribado es más importante, que el tipo de cribado utilizado (18). Durante el primer año de cribado de la retinopatía diabética, el ahorro asociado a la prevención de un solo caso de ceguera, podría cubrir el coste del programa (12).

Los programas de cribado de la retinopatía diabética en el ámbito hospitalario suelen utilizar: 1. El sistema fotográfico ETDRS. 2. La oftalmoscopia indirecta a través de pupilas dilatadas y la biomicroscopía en lámpara de hendidura $(11,13,14,16,19)$. Sin embargo, cuando es necesario acercarse al medio comunitario del enfermo los dos sistemas de cribado más utilizados son: 1 . El oftalmoscopio binocular en midriasis. 2. La realización de retinografías de $45^{\circ}$ con o sin midriasis $(11,12,19,20)$.

El uso del retinógrafo para cribado de la retinopatía diabética se considera como el método óptimo y más costo-efectivo $(1,2,11,12,21)$. La retinografía en midriasis es más sensible que la exploración con oftalmoscopia binocular. Sin embargo el cribado retinográfico masivo exige una costosa y compleja organización informática. Por ello aún en los países más desarrollados se comunican experiencias localizadas de grupos o en regiones o comunidades (1$4,15,22,23)$ y no se realiza un cribado a toda la población diabética general de un país, como se hace para tratar cualquier patología.

¿Que ocurre con nuestros pacientes con retinopatía diabética no diagnosticados?, toda exploración asume un porcentaje de error, en los retinógrafos las lesiones que quedan fuera de su campo no son detectadas. Para corregir nuestra tasa de error considera- 
mos que los exámenes anuales sucesivos en los pacientes incrementan la tasa de detección. También hemos observado como afirma Harvey (18) que la fotografía es más sensible pero que la retinopatía detectada es mayoritariamente menos grave y por tanto no precisa tratamiento. El programa ha tenido un impacto claro, aunque no cuantificado en este artículo, en la reducción del número de complicaciones de la retinopatía diabética atendidas en nuestras urgencias y en el número de vitrectomias realizadas en nuestro hospital secundarias a esta patología.

Los porcentajes de retinografías sin midriasis, no valorables, oscilan entre el $9 \%$ y el $26 \%(23,24,25)$. Este porcentaje de exploraciones no útiles se reduce del $1,4 \%$ al $8 \%$ cuando se realiza con retinógrafos de $45^{\circ}$ en midriasis farmacológica $(25,26)$. Estos enfermos no valorables necesitan un examen posterior por un oftalmólogo. En nuestra muestra con el oftalmoscopio binocular no pudimos obtener información por opacidad de medios o insuficiente midriasis en un porcentaje del 0,3\%, muy inferior a lo obtenido con la retinografía digital.

El bajo porcentaje de detección de exitus es uno de los grandes fracasos de detección del programa, el sistema sanitario no lo detecta y una parte de la falta de asistencia queda enmascarada en estos pacientes. Observamos que a mayor número de años de evolución de la diabetes, el número de pacientes que no asiste a los programas de cribado se reduce en un 3\% anual y sospechamos que la principal causa es la mortalidad.

El exitus anual esperado en una población diabética oscila entre el $2,2 \%$ anual por evento cardiovascular y alrededor el 4,22\% anual por mortalidad global (27-29). Nuestra cifra aproximada del 3\% puede ser un estimador, indirecto y poco fiable de nuestra mortalidad esperada pero en el rango que se mueve la bibliografía.

De una muestra global de 8.244 enfermos y calculando una mortalidad anual global entre el $3 \%$ y el 4,22\% implicaría que entre 247 y 339 personas habrían fallecido anualmente en el tiempo de estudio. Sin embargo, nosotros sólo recogimos seis exitus y este valor representa una cifra de alrededor del $2 \%$ de la mortalidad esperada.

Se considera un buen programa de cribado de la retinopatía diabética cuando consigue una cumplimentación y/o asistencia superior al 75\% (30). Santos Bueso (15) obtiene una participación del 80,5\% de los diabéticos censados en atención primaria. En nuestro programa el porcentaje de población que acude a la exploración es muy alta del 81,44\%. Ello puede ser debido a que nos acercamos al centro de salud del paciente, con lo que se reducen sus desplazamientos; también a la importante implicación de los médicos de familia y de la organización sanitaria. No sabemos si a largo plazo con la fatiga generada por el paso de los años, y por los inconvenientes que genera la midriasis farmacológica este porcentaje se irá reduciendo.

Desconocemos el motivo por el cual un 8,7\% de los pacientes no acudió a ninguna cita. Zoega (30) señala que existe una clara y significativa relación entre el cumplimiento de los programas de cribado de la retinopatía diabética y los resultados visuales (da cumplimentos del $77 \%$ en el grupo de buena agudeza visual y del $27 \%$ en el grupo inscrito en el registro de ciegos de Islandia). Nosotros observamos un elevado cumplimiento en nuestros pacientes siempre superior al $75 \%$ y una pequeña caída en la asistencia a medida que avanza el grado de retinopatía diabética. No creemos en nuestra muestra que esta pequeña caída en la asistencia, que pueda ser en los más avanzados de una cita de cada diez, pueda justificar una mejor o peor agudeza visual de nuestros pacientes. Creemos esta caída puede ser atribuida en parte a la edad más avanzada y a la mayor comorbilidad general que presentan los pacientes con grado de retinopatía más elevados $(14,15,27,29,31)$.

En nuestro caso creemos que la continua insistencia al diabético por medio de su médico de familia, por el endocrinólogo y por el oftalmólogo va logrando la progresiva incorporación del diabético a los programas de cribado. Sin embargo, uno de nuestros grandes fracasos es que después de 3 años de cribado continuado, aún no conocemos si a un $8,7 \%$ de nuestros pacientes diabéticos se les ha explorado alguna vez el fondo de ojo, y creemos que no ha sido así y desconocemos los motivos por los que no acudieron a su cita asignada. Otros autores aportan como causas el insuficiente conocimiento por los pacientes de las ventajas del diagnóstico y el tratamiento precoz de la retinopatía diabética, la falta de motivación y la incapacidad para acceder al cuidado oftalmológico (13).

En Galicia con un 3\% de población diabética conocida, se desea realizar un examen oftalmológico anual de fondo de ojo a todos los diabéticos conocidos (22); por lo que deberían ser explorados 30.000 pacientes por cada millón de habitantes. En nuestras tasas de prevalencia esto nos obligaría a realizar 8.244 exámenes anuales. 
El cribado de la retinopatía diabética se debe realizar en función del riesgo de pérdida de agudeza visual. Los médicos de familia americanos se marcan un objetivo tal que: el $95 \%$ de sus pacientes estén libres de retinopatía diabética, que les reste agudeza visual, en un plazo de 5 años. Para conseguir este objetivo proponen la siguiente periodicidad en los exámenes de fondo de ojo: 1 . Sin retinopatía de fondo cada 5 años. 2. Con retinopatía de fondo (leve o moderada) anual. 3. Con retinopatía preproliferativa cada 4 meses (6).

Adaptados al riesgo de los pacientes (1-3) nuestros criterios son algo más exigentes en pacientes sin retinopatía de fondo y con retinopatía moderada. Con una población asistida de 298.000 habitantes y una prevalencia de diabetes del 2,77\%. Realizamos unas 6.000 exploraciones anuales.

La fundación para la diabetes en Galicia (33) propone la realización de 48 exploraciones de fondo de ojo diarias con un retinógrafo digital, dedicando 10 minutos a cada paciente. Estas cifras son cercanas y algo inferiores a las realizadas por nosotros de 52 exploraciones/día.

El coste personal para el paciente se calcula en 46,64 euros cuando el paciente es derivado a un servicio de Oftalmología, por los gastos de desplazamiento y la pérdida de su jornada laboral (33). Pero los autores consideran que este gasto se aminora mucho cuando el enfermo es explorado en su centro de salud (33).

El coste sanitario anual de este programa es de 53.173 euros. Se considera que desplazarse a su centro de salud tiene un coste para cada enfermo de 10 Euros $(6.000 \times 10=60.000$ Euros $)$. Calculamos que el $8.3 \%$ de la población que exploramos esta activa y pierde esa jornada laboral. Si el coste fuera de unos 80 Euros/día $(6.000$ x 0,083 x $80=39.840$ euros). Los costos anuales del programa serían de unos 153.013 euros.

Cada año han sido tratados con láser 93 diabéticos detectados por el programa. Se considera que cada paciente sometido a tratamiento láser salva unos 4 años de visión (12,34). El ahorro en costos de tratar una sola persona activa detectada por el programa y considerando la renta media de España (23.000 euros) serían unos 92.000 Euros (4 x 23.000). El beneficio esperado de tratar con láser solamente a dos pacientes, en activo, con retinopatía diabética susceptible de tratamiento es superior a los costes anuales del programa.

Un oftalmólogo con un oftalmoscopio binocular $\mathrm{y}$ en midriasis puede realizar un cribado masivo a una población diabética. El programa es costoefectivo en términos económicos y sanitarios. También sirve como vehículo de educación sanitaria y a menudo permite la detección otra patología (catarata, glaucoma, degeneración macular senil, oclusión vascular) que se en nuestro caso también se derivo para su asistencia oftalmológica aunque no fuese el objetivo de este cribado.

\section{BIBLIOGRAFÍA}

1. Fernández Fernández I, Aguilar Diosdado M, Amaya Baro ML, Barrigüete Andreu MI, Benito López, P, Cornejo Castillo M, et al. Diabetes Mellitus tipo 1. Proceso asistencial integrado. Sevilla: Consejería de Salud; 2002; 1657.

2. Fernández Fernández I, Aguilar Diosdado M, Amaya Baro ML, Barrigüete Andréu MI, Benito López, P, Cornejo Castillo M, et al. Diabetes Mellitus tipo 2. Proceso asistencial integrado. Sevilla: Consejería de Salud; 2002; 1658.

3. Servicio Andaluz de Salud. Consejería de Salud. Plan integral de Diabetes. Programa de detección precoz de la retinopatía diabética. 2005; 1-26.

4. Servicio Andaluz de Salud. Consejería de Salud. Plan Integral de Diabetes. Cribado de la retinopatía diabética con retinografía digital en Atención Primaria. Test de Autoevaluación. 2005; 1-31.

5. Stefansson E. Man versus machine: Is technology a blessing or a barrier in screening for diabetic eye disease? Acta Ophthalmol Scand 2004; 82: 643-644.

6. Tubbs CG, Safeek A, Mayo HG, Markova T. Clinical inquiries. Do routine eye exams reduce occurrence of blindness from type 2 diabetes? J Fam Pract 2004; 53 : 732-734.

7. Photocoagulation for diabetic maculopathy. A randomized controlled clinical trial using the xenon arc. British Multicentre Study Group Diabetes 1983; 32: 1010-1016.

8. Wilkinson CP, Ferris FL 3rd, Klein RE, Lee PP, Agardh $C D$, Davis $M$ et al. Proposed international clinical diabetic retinopathy and diabetic macular edema disease severity scales. Ophthalmology 2003; 110: 1677-1682.

9. Fundus photographic risk factors for progression of diabetic retinopathy. ETDRS report number 12. Early Treatment Diabetic Retinopathy Study Research Group Ophthalmology 1991; 98: 823-833.

10. López-Gálvez MI. Escala internacional de severidad de la retinopatía y del edema macular diabético. Arch Soc Esp Oftalmol 2004; 79: 149-150.

11. Fong DS, Aiello LP, Ferris FL 3rd, Klein R. Diabetic retinopathy. Diabetes Care 2004; 27: 2540-2553.

12. Faria-Abreu JR. Epidemiología. In: Cunha-Vaz J. Retinopatía diabética. Madrid: MacLine. $1^{a}$ ed. 2006; 173-202.

13. Bonafonte S, García CA, Davis MD. Clasificación de la retinopatía diabética y el edema macular. In: Bonafonte $S$. Retinopatía diabética. Madrid: Elsevier. $2^{a}$ ed. 2006; 73-99.

14. Teruel-Maicas C, Fernández-Real JM, Ricart W, Valent Ferrer $R$, Vallés Prats $M$. Prevalencia de la retinopatía diabética en la población de diabéticos diagnosticados en 
las comarcas de Girona. Estudio de los factores asociados. Arch Soc Esp Oftalmol 2005; 80: 85-91.

15. Santos-Bueso E, Fernández-Vigo J, Fernández Pérez, C, Macarro Merino A, Fernández Perianes J. Prevalencia de retinopatía diabética en la comunidad Autónoma de Extremadura. 1997-2001 (Proyecto Extremadura para prevención de la ceguera). Arch Soc Esp Oftalmol 2005; 80: 187-194.

16. Romero-Aroca P, Fernández-Alart J, Baget-Bernaldiz M, Méndez-Marín I, Salvat-Serra M. Epidemiología de la retinopatía diabética en pacientes tipo II. Cambios observados en una población entre los años 1993 y 2005, tras los nuevos criterios diagnósticos y un mayor control de los pacientes. Arch Soc Esp Oftalmol 2007; 82: 209-218.

17. Kempen JH, $O^{\prime}$ Colmain BJ, Leske MC, Haffner SM, Klein $R$, Moss SE et al. The prevalence of diabetic retinopathy among adults in the United States. Arch Ophthalmol 2004; 122: 552-563.

18. Harvey JN, Craney L, Nagendran S, Ng CS. Towards comprehensive population-based screening for diabetic retinopathy: operation of the North Wales diabetic retinopathy screening programme using a central patient register and various screening methods. J Med Screen 2006; 13: 87-92.

19. Lopez-Bastida J, Cabrera-Lopez F, Serrano-Aguilar P. Sensitivity and specificity of digital retinal imaging for screening diabetic retinopathy. Diabet Med 2007; 24: 403-407.

20. Agarwal S, Mahajan S, Rani PK, Raman R, Paul PG, Kumaramanickavel $G$, et al. How high is the non-response rate of patients referred for eye examination from diabetic screening camps?. Ophthalmic Epidemiol 2005; 12: 393-394.

21. Sinclair SH. Diabetic retinopathy: the unmet needs for screening and a review of potential solutions. Expert Rev Med Dev 2006; 3: 301-313.

22. Henahan $S$. Remote diabetic retinopathy screening reaches those most at risk. Eurotimes 2007; 12: 10.

23. Soulie-Strougar M, Charles A, Metral P, Quercia P, Souchier $M$, Chirpaz $L$, et al. Dépistage de la rétinopathie diabétique en Bourgogne par un rétinographe non mydriatique itinerant. J Fr Opthalmol 2007; 30: 121-126.
24. Boucher MC, Nguyen QT, Angioi K. Mass community screening for diabetic retinopathy using a nonmydriatic camera with telemedicine. Can J Ophthalmol 2005; 40: 734-742.

25. Murgatroyd H, Ellingford A, Cox A, Binnie M, Ellis JD, MacEwen CJ et al. Effect of mydriasis and different field strategies on digital image screening of diabetic eye disease. Br J Ophthalmol 2004; 88: 920-924.

26. Saari JM, Summanen P, Kivela T, Saari KM. Sensitivity and specificity of digital retinal images in grading diabetic retinopathy. Acta Ophthalmol Scand 2004; 82: 126130.

27. Knudtson MD, Klein BE, Klein R. Age-related eye disease, visual impairment, and survival: the Beaver Dam Eye Study. Arch Ophthalmol 2006; 124: 243-249.

28. Swenson CJ, Trepka MJ, Rewers MJ, Scarbro S, Hiatt WR, Hamman RF. Cardiovascular disease mortality in Hispanics and non-Hispanic whites. Am J Epidemiol 2002; 156:919-928.

29. Gimeno Orna JA, Boned Juliani B, Lou Arnal LM, Castro Alonso FJ. Mortalidad en una cohorte de pacientes con diabetes tipo 2 del área de Alcañiz, España. An Med Interna 2002; 19: 336-340.

30. Zoega GM, Gunnarsdottir T, Bjornsdottir S, Hreietharsson AB, Viggosson G, Stefansson E. Screening compliance and visual outcome in diabetes. Acta Ophthalmol Scand 2005; 83: 687-690.

31. Esteban Ortega MM, Rodríguez Hurtado F, Jiménez. Moleón JJ, Bueno Cavanillas A. Prevalencia de la retinopatía en diabéticos de más de diez años de evolución en la zona Norte de Granada. Arch Soc Esp Oftalmol 1999; 74: 137-144.

32. González F, López MI, Gómez-Ulla F. La teleoftalmología en la retinopatía diabética. In: Alfaro $V$, Gómez-Ulla F, Quiroz-Mercado H, Suárez-Figueroa M, Villalba SJ. Retinopatía Diabética. Tratado médico quirúrgico. Madrid: MacLine. 2006: 105-111.

33. Arana $R$. El coste de no prevenir. Infodiab 2004; 25: 1.

34. Javitt JC, Aiello LP. Cost-effectiveness of detecting and treating diabetic retinopathy. Ann Inter Med 1996; 124: 164-169. 